\title{
A UNIQUENESS RESULT IN CONFORMAL MAPPING. II
}

\author{
JAMES A. JENKINS ${ }^{1}$
}

\begin{abstract}
This paper gives an elementary proof of the result that for a function $f$ in the family $\Sigma$ the diameter of the complement of the image of $|z|>1$ by $w=f(z)$ attains its minimal value 2 only for $f(z)=z+c, c$ constant.
\end{abstract}

1. Some years ago the author gave the first published proof [2] of the result that if, for a function $f$ in the family $\Sigma[1]$, the diameter of the complement of the image of $|z|>1$ by $w=f(z)$ attains its minimal value 2 , then $f(z)=z+c$ ( $c$ constant). While the proof is very simple, it utilizes results and concepts which would not normally be familiar to a student in a good basic course in Function Theory, which is the natural context for the above result. More recently Pfluger $[3,4]$ has given several versions of a proof of this result which seem to him to be more elementary. It appears that they also would require some digression from the material usually found in a Function Theory course. Our purpose here is to give an elementary proof in purely Function Theoretic terms.

2. LEMMA 1. Let $f \in \Sigma$ have Laurent expansion about the point at infinity

$$
f(z)=z+c_{0}+\sum_{n=1}^{\infty} c_{n} z^{-n}
$$

Let the complement of the image of $|z|>1$ under $w=f(z)$ have diameter $D$. Then $D \geq 2$ and a necessary condition for equality is $c_{2 n+1}=0, n$ integral $\geq 0$.

Let $D_{r}$ be the diameter of the image of $|z|=r, r>1$, under $w=f(z) ; D_{r}^{*}=$ $\max _{|z|=r}|f(z)-f(-z)|$. Evidently $D_{r} \geq D_{r}^{*} \geq D$ and $\lim _{r \rightarrow 1} D_{r}=D$. Further

$$
\left(D_{r}^{*}\right)^{2} \geq \frac{1}{2 \pi} \int_{0}^{2 \pi}\left|f\left(r e^{i \theta}\right)-f\left(-r e^{i \theta}\right)\right|^{2} d \theta=4 r^{2}+4 \sum_{n=0}^{\infty}\left|c_{2 n+1}\right|^{2} r^{-2(2 n+1)} .
$$

Thus $D \geq 2$ with equality only if $c_{2 n+1}=0, n=0,1,2, \ldots$.

The following lemma is found in [3].

LEMMA 2. Using the same notation as in Lemma 1, $D_{r} \leq r D, r>1$.

Let $\left|z_{1}\right|=\left|z_{2}\right|=r, r>1$, be such that $\left|f\left(z_{1}\right)-f\left(z_{2}\right)\right|=D_{r}$ and let $\Phi(z)=$ $f\left(z_{1} z_{2}^{-1} z\right)-f(z)$. Then for $\rho>1, \max _{|z|=\rho}|\Phi(z)| \leq D_{\rho}$. The function $z^{-1} \Phi(z)$ is regular outside the unit circle (including the point at infinity) thus $\left|z^{-1} \Phi(z)\right| \leq$ $\rho^{-1} D_{\rho}$ in $|z| \geq \rho$. Letting $\rho$ tend to 1 we have $\left|z^{-1} \Phi(z)\right| \leq D$ in $|z|>1$. Thus $D_{r} \leq r D$.

Received by the editors May 14, 1981.

1980 Mathematics Subject Classification. Primary 30C25, 30C55, 30C75.

${ }^{1}$ Research supported in part by the National Science Foundation.

(c) 1982 American Mathematical Society 0002-9939/81/0000-0300/\$01.50 
THEOREM. Let $f \in \Sigma$ and let the complement of the image of $|z|>1$ under $w=f(z)$ have diameter $D$. Then $D \geq 2$ and $D=2$ if and only if $f(z)=z+c, c$ constant.

It remains only to consider the possibility $D=2$. If $f(z)$ is not $z+c$ let it have the Laurent expansion (1). By Lemma 1 the first nonzero coefficient beyond the constant term would be $c_{2 n}, n \geq 1$. By Lemmas 1 and $2, D_{r}=D_{r}^{*}=2 r$ thus $\left|f\left(r e^{i \theta}\right)-f\left(r e^{i \varphi}\right)\right|^{2}$ would be maximized for $\varphi=\theta+\pi$ for all $r>1, \theta$ real and the partial derivative of this quantity with respect to $\varphi$ evaluated at $\varphi=\theta+\pi$ would be identically zero in this set. Inserting the expansion (1) for $f$ the coefficient of $r^{-2 n+1}$ would be

$$
4 n i\left(c_{2 n} e^{-(2 n+1) i \theta}-\bar{c}_{2 n} e^{(2 n+1) i \theta}\right)
$$

which would imply the contradiction $c_{2 n}=0$.

\section{REFERENCES}

1. James A. Jenkins, Univalent functions and conformal mapping, Springer-Verlag, BerlinGöttingen-Heidelberg, 1958.

2.

3. A. Pfluger, On a uniqueness theorem in conformal mapping, Michigan Math. J. 23 (1976), 363365.

4. $\ldots$, On the diameter of planar curves and Fourier coefficients, J. Appl. Math. Phys. 30 (1979), 305-314.

School of Mathematics, The institute for Advanced Study, Princeton, NEW JERSEY 08540

Current address: Department of Mathematics, Washington University, St. Louis, Missouri 63130 